\title{
GAIA Level 3 Antepartum Stillbirth
}

National Cancer Institute

\section{Source}

National Cancer Institute. GAIA Level 3 Antepartum Stillbirth. NCI Thesaurus. Code C128020.

GAIA Level 3 Antepartum Stillbirth is defined by three criteria: first, delivery of an infant with no signs of life at birth, including the following: no spontaneous movements, no umbilical cord pulse, no heartbeat, no crying, no spontaneous respirations or chest movement, and full body cyanosis; second, one or more of the following three requirements must be met: a) Maternal report of the absence of fetal movement for 24 hours or more prior to delivery; b) Report of auscultation for fetal heart tones using electronic devices (e.g. handheld Doppler or fetal heart rate monitor) or non-electronic devices (e.g. stethoscope, fetoscope or Pinard horn) that documents the absence of a fetal heartbeat; c) Non-attended delivery followed by a post-delivery physical examination of the fetus that is performed by a health care professional who meets the standard of care in the immediate health care setting, and that is consistent with antepartum death; d) a verbal history provided by a trained health care provider, non-medical witness, or the mother of the fetus documenting that the fetus was born with no signs of life, was unresponsive to resuscitation efforts immediately after delivery, and had physical features consistent with antepartum death; third, gestational age within the pre-defined range for selected stillbirth definition as assessed by maternal and/or fetal parameters. 